\title{
Searching for spiral features in the outer Galactic disk ${ }^{\star}$ The field towards WR38 and WR38a
}

\author{
G. Carraro ${ }^{1,2}$ and E. Costa ${ }^{3}$ \\ 1 ESO, Casilla 19001, Santiago, Chile \\ e-mail: gcarraro@eso.org \\ 2 Dipartimento di Astronomia, Università di Padova, Vicolo Osservatorio 2, 35122 Padova, Italy \\ 3 Departamento de Astronomía, Universidad de Chile, Casilla 36-D, Santiago, Chile
}

Received 13 August 2008 / Accepted 3 October 2008

ABSTRACT

\begin{abstract}
Context. The detailed spiral structure of the outer Galactic disk remains poorly constrained, and for several line of sights in the Galaxy we depend on model extrapolations.

Aims. One of these regions is the fourth Galactic quadrant, between Vela and Carina $\left(270^{\circ} \leq l \leq 300^{\circ}\right)$, where, apart from the conspicuous Carina branch of the Carina Sagittarius arm, no spiral arms have been detected so far in the optical beyond $l \sim 270^{\circ}$.

Methods. By means of deep UBVI photometry, we search for spiral features in known low absorption windows. Although observationally demanding, $U$ photometry constitutes a powerful tool for detecting and characterizing distant aggregates of young stars, and allows firmer distance estimates to be derived. We study a direction close to the tangent $\left(l \sim 290^{\circ}\right)$ of the Carina arm, in an attempt to detect optical spiral tracers far beyond the Carina branch, where radio observations and model predictions appear to indicate the presence of an extension of the Perseus and Norma-Cygnus spiral arms in the fourth quadrant.

Results. Along this line of sight, we detect three distinct groups of young stars. Two of them, at distances of $\sim 2.5$ and $\sim 6.0 \mathrm{kpc}$, belong to the Carina spiral arm, which is traversed twice in this particular direction. Interestingly, the latter is detected for the first time. The third group, at a distance of $\sim 12.7 \mathrm{kpc}$, is probably related to the Perseus arm which lies beyond the Carina arm, and constitutes the first optical detection of this arm in the fourth Galactic quadrant. The position of this feature is consistent with both HI observations and model predictions. We also present evidence that this extremely distant group, formerly assumed to be a star cluster (Shorlin 1), is a diffuse, young population, typically found in spiral galaxies. In addition, our data-set does not support, as claimed in the literature, the possible presence of the Monoceros Ring in this direction

Conclusions. This study highlights how multicolor optical studies can be effective in probing the spiral structure of the outer Galactic disk. More fields need to be studied in this region of the Galaxy to constrain the spiral structure in the fourth Galactic quadrant more accurately, in particular, the shape and extent of the Perseus arm, and, possibly, to detect the even more distant Norma-Cygnus arm.
\end{abstract}

Key words. Galaxy: general - Galaxy: structure - Galaxy: stellar content

\section{Introduction}

The study of the spiral structure in the outer Galactic disk has experienced a renewed interest (see e.g. Levine et al. 2006; Vázquez et al. 2008; and Benjamin 2008), in part due to the claimed discovery of structures in the form of stellar overdensities which could be produced by accretion/merging events. Examples are those in Monoceros (Yanny et al. 2003), Canis Major (Martin et al. 2004), and Argus (Rocha Pinto et al. 2006). To assess the reality and properties of these overdensities, we must complete a detailed investigation of the outer Galactic disk. This investigation would also improve our knowledge of the extreme periphery of the Milky Way (MW).

Spiral features can be detected by using a variety of tracers, namely HI (e.g. Levine et al. 2006), HII (e.g. Russeil 2003), CO (see e.g. Luna et al. 2006), and optical objects (e.g. Vázquez et al. 2008). These different techniques have provided a complete picture of the spiral structure in the third Galactic quadrant (Moitinho et al. 2008). Our group contributed to this

\footnotetext{
* Photometric catalog is only available in electronic form at the CDS via anonymous ftp to cdsarc.u-strasbg. fr (130.79.128.5) or via http://cdsweb.u-strasbg.fr/cgi-bin/qcat?J/A+A/493/71
}

effort by collating optical data sets for a large sample of young open clusters (Moitinho et al. 2006), in the field of which we recognized distant, reddened young aggregates, which allowed us to define the shape and extent of spiral arms out to $20 \mathrm{kpc}$ from the Galactic center.

This paper presents the first results of a study of selected low absorption regions in the fourth Galactic quadrant, which aims to identify distant optical spiral features. We investigate the field towards the Wolf-Rayet (WR) stars WR38 and WR38a $\left(\mathrm{RA}=11^{\mathrm{h}} 06^{\mathrm{m}}\right.$, Dec $=-61^{\circ} 14^{\prime}$, Shorlin et al. 2004, hereafter Sh04; Wallace et al. 2005, hereafter Wa05), which attracted our attention for several reasons.

Sh04 and Wa05 presented the discovery of a distant, young, compact star cluster, at a distance of $\sim 12 \mathrm{kpc}$, associated with these two WR stars. The two studies, however, reached quite different conclusions about the relationship between this cluster and known spiral arms. While Sh04 proposed that the cluster is associated with an extension of the Perseus arm beyond the Carina-Sagittarius arm, Wa05, more conservatively, suggested that it is located in the more distant part of the Carina arm. The line-of-sight to WR38 and WR38a does indeed cross the 


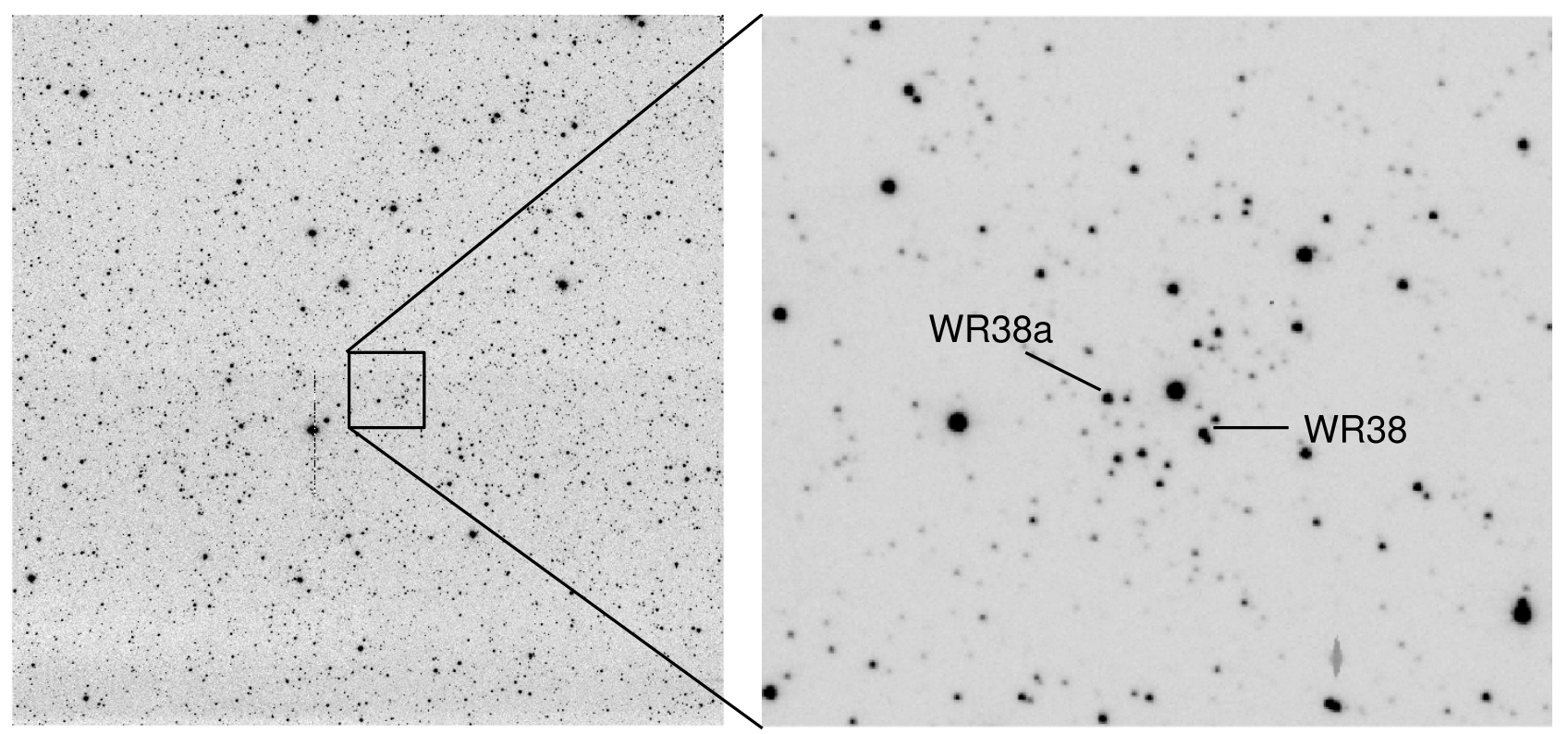

Fig. 1. Left panel: $V$-band $100 \mathrm{~s}$ exposure image of the field observed in the area of the star cluster Shorlin 1. Field shown is $20^{\prime}$ on a side. North is up and East to the left. Right panel: zoom of the cluster region, in which the two WR stars (WR38 and WR38a) are indicated. Field shown is $~ 3^{\prime}$ on a side.

Carina-Sagittarius arm twice. With a quite different interpretation, Frinchaboy et al. (2004) associated this cluster with the Galactic Anticenter Stellar Structure (GASS), a population of old star clusters, possibly members of the Monoceros Ring.

To help clarifying these issues, we undertook an observational campaign to address the following questions: is the group of stars close to WR38 and WR38a (i.e. Shorlin 1) a real star cluster? Does this group belong to the most distant part of the Carina arm or to the Perseus arm? Are we looking in this direction at signatures of the Monoceros ring, or the Argus system?

The paper is organized as follows. In Sect. 2, we describe the observations and data reduction procedure and compare our data set with previous investigations, and, in Sect. 3, we discuss the reddening law in the Galactic direction under consideration. In Sects. 4 and 5 we discuss in detail the properties of the three different stellar populations detected in the field observed. Finally, in Sect. 6 we summarize the results of our study.

\section{Observations and data reduction}

\subsection{Observations}

The region of interest (see Fig. 1) was observed with the Y4KCAM camera attached to the $1.0 \mathrm{~m}$ telescope, which is operated by the SMARTS consortium ${ }^{1}$ and located at Cerro Tololo Inter-American Observatory (CTIO). This camera is equipped with an STA $4064 \times 4064$ CCD with $15-\mu$ pixels, yielding a scale of $0.289^{\prime \prime} /$ pixel and a field-of-view (FOV) of $20^{\prime} \times 20^{\prime}$ at the Cassegrain focus of the CTIO $1.0 \mathrm{~m}$ telescope. The CCD was operated without binning, at a nominal gain of $1.44 \mathrm{e}^{-} / \mathrm{ADU}$, implying a readout noise of $7 \mathrm{e}^{-}$per quadrant (this detector is read by means of four different amplifiers). QE and other detector characteristics can be found $\mathrm{at}^{2}$.

The observational data were acquired in three observing runs, as summarized in Table 1. In our first run we obtained

\footnotetext{
1 http://http://www. astro.yale.edu/smarts

2 http://WWW . astronomy . ohio-state . edu/Y4KCam/ detector .html
}

Table 1. Log of $U B V I$ photometric observations.

\begin{tabular}{lcccc}
\hline \hline Target & Date & Filter & Exposure (s) & airmass \\
\hline Shorlin 1 & 24 May 2006 & $U$ & 60,1500 & $1.28-1.33$ \\
& & $B$ & $30,100,1200$ & $1.20-1.35$ \\
& & $V$ & $30,100,900$ & $1.22-1.34$ \\
\multirow{4}{*}{ Shorlin 1 29 January 2008 } & $I$ & 100,700 & $1.25-1.34$ \\
& & $B$ & 5,15 & $1.19-1.22$ \\
& & $V$ & $3,5,10$ & $1.19-1.23$ \\
SA 98 & 29 January 2008 & $U$ & $2 \times 10,200,300,400$ & $1.17-1.23$ \\
& & $B$ & $2 \times 10,100,2 \times 200$ & $1.17-1.99$ \\
& & $V$ & $2 \times 10,25,50,2 \times 100$ & $1.20-2.27$ \\
Shorlin 1 & 05 June 2008 & $U$ & $2 \times 10,50,100,2 \times 150$ & $1.18-2.05$ \\
& & $U$ & $30 \times 3,1800$ & $1.19-1.20$ \\
\hline
\end{tabular}

deep $U B V I$ images of Shorlin 1, under good seeing, but nonphotometric conditions. In our second run, we took medium and short exposures of Shorlin 1, and observed Landolt's SA 98 UBVRI standard stars area (Landolt 1992), to be able to relate our $U B V I$ instrumental system to the standard system. In a final third run, we secured an additional set of $U$-band images of Shorlin 1. Average seeing was throughout all runs $1.2^{\prime \prime}$.

Our $U B V I$ instrumental photometric system was defined by the use of a standard broad-band Kitt Peak $B V R_{k c} I_{k c}$ set in combination with a $\mathrm{U}+\mathrm{CuSO} 4 U$-band filter. Transmission curves for these filters can be found $\mathrm{at}^{3}$. To determine the transformation from our instrumental system to the standard JohnsonKron-Cousins system, we observed 46 stars in area SA 98 (Landolt 1992) multiple times, and with different airmasses ranging from $\sim 1.2$ to $\sim 2.3$. Field SA 98 is very advantageous, as it includes a large number of well observed standard stars, and it is completely covered by the CCD's FOV. Furthermore, the standard's color coverage is very good, being: $-0.5 \leq(U-B) \leq 2.2$; $-0.2 \leq(B-V) \leq 2.2$ and $-0.1 \leq(V-I) \leq 6.0$.

\footnotetext{
${ }^{3}$ http://www . astronomy. ohio-state. edu/Y4KCam/filters. html
} 


\subsection{Data reduction}

Basic calibration of the CCD frames was done using the IRAF package CCDRED. For this purpose, zero-exposure frames and twilight sky flats were acquired every night. Photometry was then performed using the IRAF DAOPHOT and PHOTCAL packages. Instrumental magnitudes were extracted following the point spread function (PSF) method (Stetson 1987). A quadratic, spatially variable, Master PSF (PENNY function) was adopted. The PSF photometry was finally aperture-corrected, filter by filter. Aperture corrections were determined by performing aperture photometry for a suitable number (typically 10 to 20) of bright stars in the field. These corrections were found to vary between 0.120 and $0.215 \mathrm{mag}$, depending on the filter.

\subsection{The photometry}

Our final photometric catalog consists of 7425 entries with $U B V I$ measurements down to $V \sim 20$, and 12250 entries having $B V I$ measures down to $V \sim 22$.

After removing both saturated stars and stars having only a few measurements in the catalog of Landolt's (1992), our photometric solutions for a grand total of 183 measurements in $U$ and $B$, and of 206 measurements in $V$ and $I$, are given by:

$$
\begin{aligned}
U=u & +(3.290 \pm 0.010)+(0.47 \pm 0.01) \\
& \times X-(0.010 \pm 0.016) \times(U-B) \\
B= & b+(2.220 \pm 0.012)+(0.29 \pm 0.01) \\
& \times X-(0.131 \pm 0.012) \times(B-V) \\
V= & +(1.990 \pm 0.007)+(0.16 \pm 0.01) \\
& \times X+(0.021 \pm 0.007) \times(B-V) \\
I= & i+(2.783 \pm 0.011)+(0.08 \pm 0.01) \\
& \times X+(0.043 \pm 0.008) \times(V-I) .
\end{aligned}
$$

The final rms of the fitting was $0.073,0.069,0.035$, and 0.030 in $U, B, V$, and $I$, respectively.

Global photometric errors were estimated using the scheme developed by Patat \& Carraro (2001, Appendix A1), which takes account of the errors in the PSF fitting procedure (i.e. from ALLSTAR), and the calibration errors (corresponding to the zero point, color terms, and extinction errors). In Fig. 2, we present global photometric error trends plotted as a function of $V$ magnitude. Quick inspection indicatess that stars brighter than $V \approx 20$ mag have errors lower than $0.10 \mathrm{mag}$ in magnitude and lower than $0.20 \mathrm{mag}$ in all colors.

The only previous $U B V$ (Johnson-Morgan) photometric study of this region was that of Sh04, who presented photoelectric and CCD photometry for approximately 140 stars brighter than $V \sim 16.5$ in the field of WR38 and WR38a. In Fig. 3, we compare our photometry with that of Sh04 for $V, B-V$, and $U-B$, by subtracting their results from our own, as a function of our $V$ magnitude. This comparison was possible only for 103 of their stars because, from the material presented in Sh04, it was not possible to identify all objects measured by them (this issue was discussed with Turner, private communication). From the stars in common we obtain: $\Delta V=0.017 \pm 0.065$, $\Delta(B-V)=0.014 \pm 0.066$, and $\Delta(U-B)=0.034 \pm 0.199$.

In spite of the significant scatter in the comparison for $U-B$, it can be concluded that the two studies are in good agreement.

\footnotetext{
4 IRAF is distributed by the National Optical Astronomy Observatory, which is operated by the Association of Universities for Research in Astronomy, Inc., under cooperative agreement with the National Science Foundation.
}

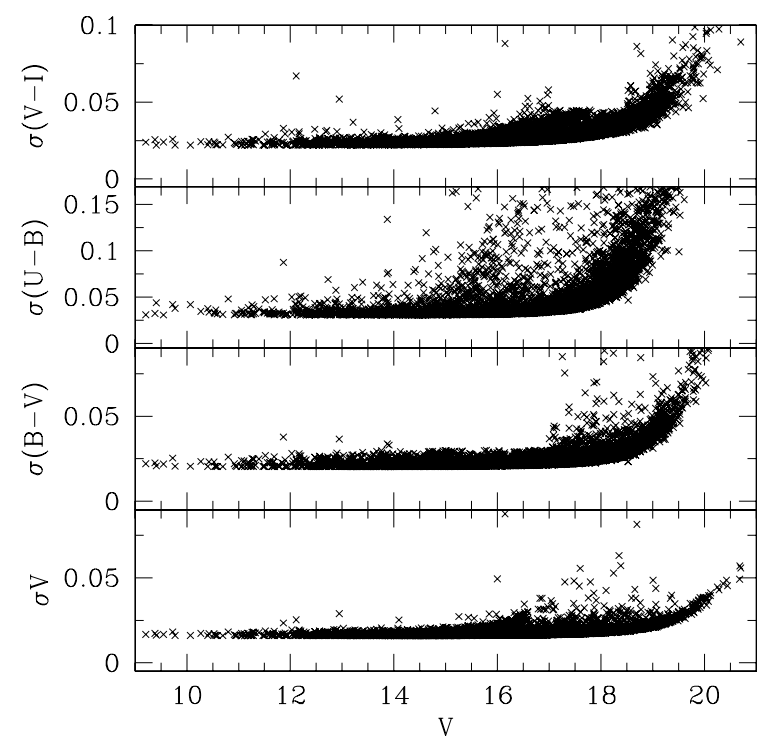

Fig. 2. Photometric errors in $V,(B-V),(U-B)$ and $(V-I)$ as a function of $V$ mag.

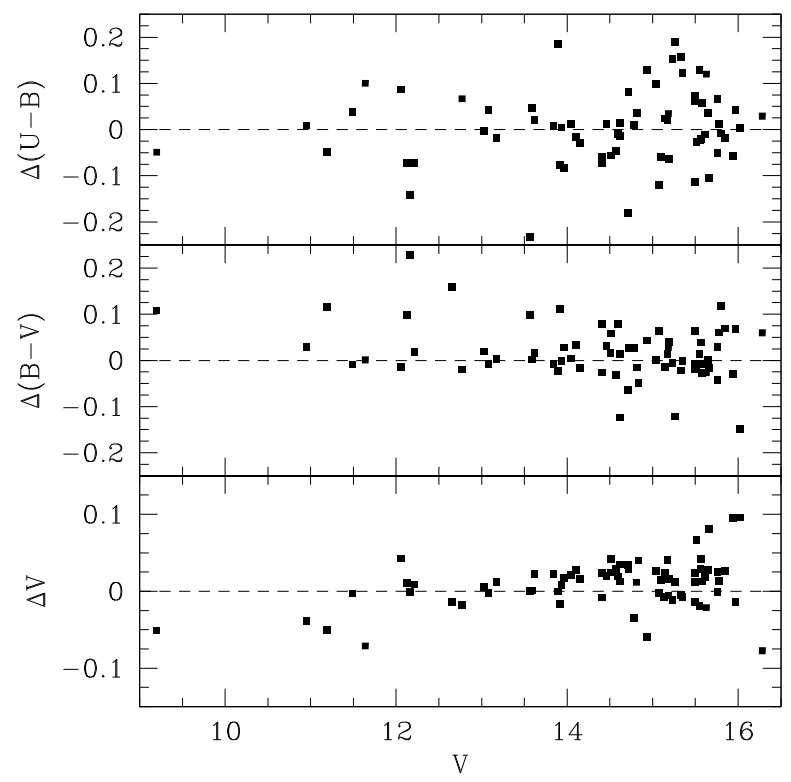

Fig. 3. Comparison of our photometry with that of Sh04 for $V, B-V$, and $U-B$ (such that our work is presented after subtraction of the results of Sh04), as a function of our $V$ magnitude.

The poor agrrement for this color could be attribed to problems in their $U$-band photometry, as extensively discussed by Sh04. We therefore also confirmed that the discrepancies between the photometry Sh04 and of Wa05 were possibly due to the transformation of the F336 $(U)$, F439W $(B)$, and F555W $(V)$ HST photometry to the Johnson-Morgan system.

\subsection{Astrometry}

For approximately two-hundred stars in our photometric cata$\log$ J2000.0 equatorial coordinates are available from the Guide Star Catalogue $e^{5}$, version 2 (GSC-2.2, 2001). Using the SkyCat tool at ESO and the IRAF tasks ccxymatch and ccmap, we first

\footnotetext{
5 Space telescope Science Institute, 2001, The Guide Star Catalogue Version 2.2.02.
} 


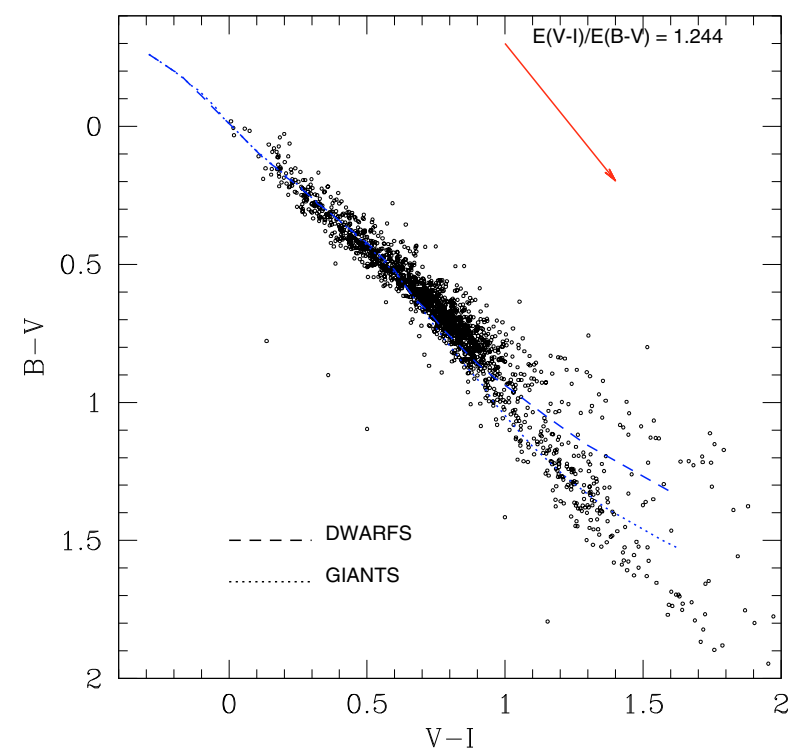

Fig. 4. $(V-I)$ vs. $(B-V)$ color-color diagram for stars in our field with $U B V I$ photometry. The reddening vector for the normal extinction law is plotted in the upper-right corner. The dashed and dotted lines represent the reddening-free Schmidt-Kaler (1982) Zero Age Main Sequence relations for dwarf and giant stars, respectively.

established a transformation between our $(X, Y)$ pixel coordinates (from ALLSTAR) and the International Celestial Reference Frame (Arias et al. 1995). These transformations have an rms value of typically $0.15^{\prime \prime}$. Finally, using the IRAF task cctran, we computed J2000.0 coordinates for all objects in our catalog.

\section{The reddening law toward $I=290.63$ and $b=-0.903$}

Before analyzing our photometric data, a basic requirement is to investigate the reddening law in the direction of WR38 and WR38a. According to their analysis, Sh04 emphasized that the extinction in this direction followed the normal law, namely that the ratio of total to selective absorption $\frac{A_{V}}{E(B-V)}$ is 3.1 . This value was already found to be in general valid towards Carina (Carraro 2002; Tapia et al. 2003). The $(V-I)$ versus $(B-V)$ color-color diagram shown in Fig. 4, which was constructed using our $U B V I$ photometry, confirms the above statements. Most stars are located close to the reddening-free Schmidt-Kaler (1982) Zero Age Main Sequence (ZAMS) line (dashed line), which runs almost parallel to the reddening vector plotted in the upper-right corner of this figure. This vector has been drawn following the normal extinction ratio (Dean et al. 1978), which suggests that, in this specific Galactic direction, absorption follows the normal law. In the subsequent discussion, we will therefore adopt $\frac{A_{V}}{E(B-V)}=3.1$ and $\frac{E(U-B)}{E(B-V)}=0.72$.

\section{Stellar populations in the field}

In Fig. 5, we present a $(B-V)$ versus $(U-B)$ color-color diagram for those stars in our photometric catalog, which have photometric errors lower than $0.05 \mathrm{mag}$ in both $(B-V)$ and $(U-B)$. This figure is similar to Fig. 3 of Sh04, apart for the larger number of stars due to our larger area coverage and deeper photometry. The solid line is an empirical reddening-free ZAMS for dwarf stars, from Schmidt-Kaler (1982). In agreement with the discussion in the previous section, we have adopted a normal reddening law for this region. The corresponding reddening vector has been plotted in the bottom of the figure.

Shifting the ZAMS by different amounts in the direction of the reddening vector, we can fit the most obvious stellar sequences, which has led us to identify three distinct stellar populations. This displacement is illustrated by dotted lines (in black) for three spectral type along the reddening direction. This permits us to define the spectral type of reddened stars.

We now discuss why we have chosen these amounts and claim that we are distinguishing three different populations.

The yellow dot-dashed ZAMS has been shifted by $E(B-$ $V)=0.25$, and fits a conspicuous sequence of early type stars. As indicated in the figure, this group consists of stars of spectral type as early as $\mathrm{O} 8$ to A0. Beyond A0, the definition of spectral type becomes ambiguous due to the crossing of different reddening ZAMS close to the A0V knee. This low reddening group of stars clearly suffers from variable extinction, as indicated by the significant spread about the $E(B-V)=0.25$ ZAMS. We estimate in fact that the mean reddening for this group is $0.25 \pm 0.10$. Sh04 identified this group as stars belonging to the Carina branch of the Carina-Sagittarius spiral arm. We will refer to this group of stars as group $\mathbf{A}$.

The blue short-dashed ZAMS has been shifted by $E(B-V)=$ 0.52 . It passes through a group of young stars, of spectral types ranging from $\mathrm{B} 2$ to $\mathrm{A} 0$, which exhibit almost no reddening variation. In fact, we estimate that the mean reddening for this group is $0.52 \pm 0.05$, namely the scatter about the mean value of 0.52 is small, and compatible with the typical photometric error in the $(U-B)$ and $(B-V)$ colors. In Fig. 3 of Sh04 this group is hardly detectable, and the authors offer no comments about the group. Because of the wider area covered by our study, it could be readily detected in our $(B-V)$ vs. $(U-B)$ color-color diagram. We refer to this group of stars as group $\mathbf{B}$.

Similar features (namely secondary sequences in two-color diagrams) have been found in a variety of two-color diagrams of stellar clusters and field stars in the third Galactic Quadrant (see e.g. Carraro et al. 2005). They are interpreted as being produced by early-type stars belonging to distant spiral features in the outer Galactic disk (see also Moitinho et al. 2008).

The two red long-dash ZAMSs have been shifted by $E(B-$ $V)=1.10$ and $E(B-V)=1.50$, and appear to isolate a group of early-type (from O6 to B5) stars that hace experienced high amount of reddening with a significant dispersion. Since the region between the two red ZAMS in the color-color diagram is contigouously occupied by stars, we propose that these earlytype stars basically belong to the same distant population.

We estimate that the mean reddening of this group is $1.30 \pm 0.20$.

Within this group of stars, Sh04 identified a cluster of young stars surrounding the two WR stars indicated by the two crosses in Fig. 5. We refer to this group of stars as group $\mathbf{C}$ or Shorlin 1 (also known as C1104-610a).

The two crosses in Fig. 5 identify the two WR stars, WR38 (WC4) and WR38a (WN5). As discussed by Sh04, U-band photometry of this type of star is difficult, but they do provide corrections for transforming their colors to the Johnson-Morgan standard system. Given that our uncorrected colors (see Table 2) are in good agreement with theirs, we adopted their corrections (see Table 3 in Sh04).

\section{Properties of groups A, B, and C}

To be more quantitative, we first measured the individual reddening of the stars in groups $\mathbf{A}, \mathbf{B}$, and $\mathbf{C}$ using the well known 


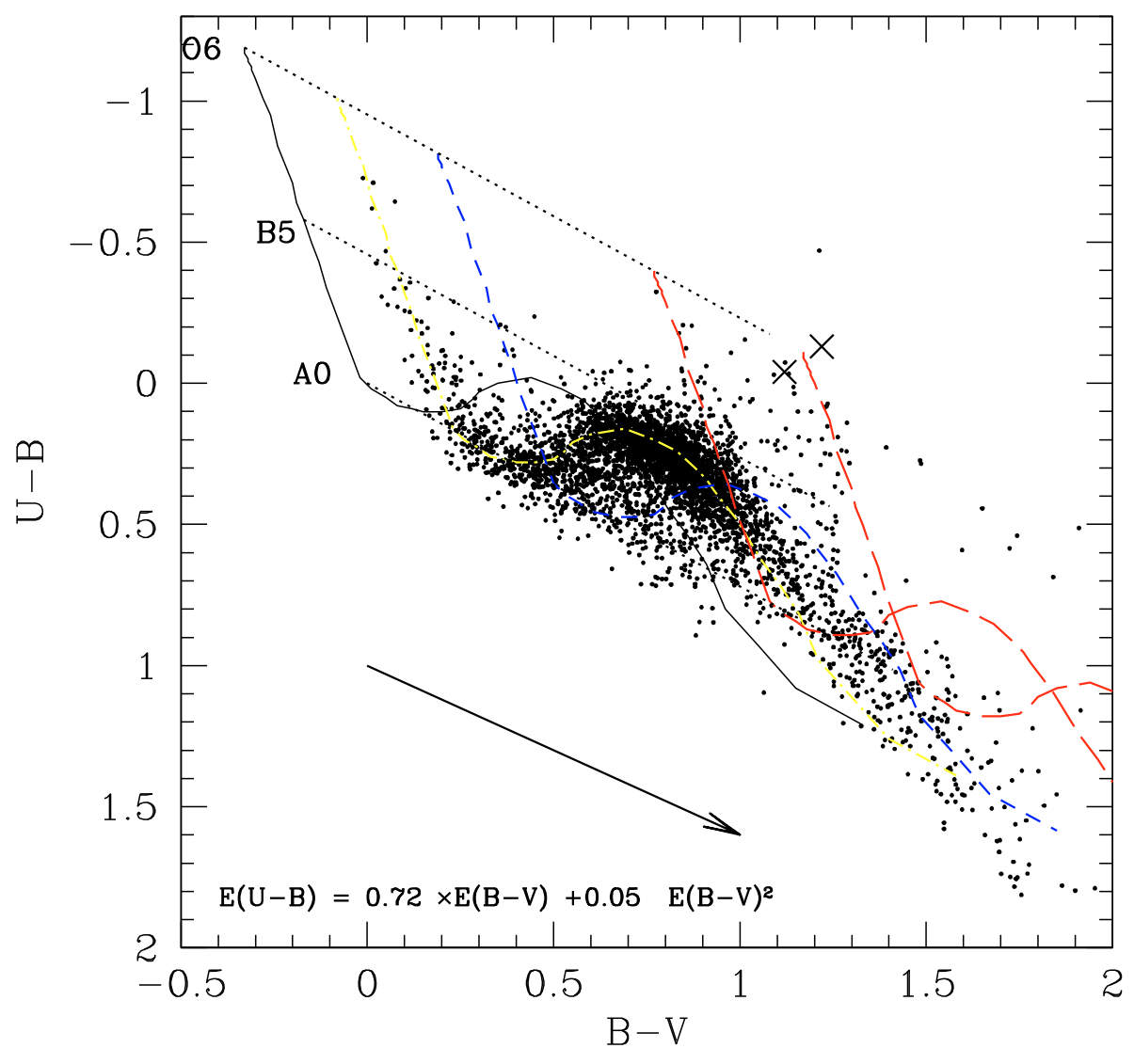

Fig. 5. $(B-V)$ vs. $(U-B)$ color-color diagram for stars in our photometric catalog with photometric errors lower than 0.05 mag in both $(B-V)$ and $(U-B)$. The reddening vector for the normal extinction law has been plotted in the bottom of the figure. The solid line is an empirical reddening-free ZAMS for dwarf stars, from Schmidt-Kaler (1982). The same ZAMS (dashed lines) has been displaced along the reddening line for three different amounts of reddening, $0.25,0.52$, and 1.45 . The approximate location of stars with spectral type O6, B5, and $\mathrm{A} 0$ is also indicated. The two crosses are used to indicate the position of WR38 and WR38a. See text for more details.
Table 2. Photometry of the stars WR38 and WR38a.

\begin{tabular}{lccc}
\hline \hline & $V$ & $(B-V)$ & $(U-B)$ \\
\hline WR38 & 14.684 & 1.216 & 0.679 \\
WR38a & 15.113 & 1.191 & 0.077 \\
\hline
\end{tabular}

reddening free $Q$-parameter (see for instance Johnson 1966), which allows us to establish the membership of a particular star to a group on the basis of common reddening (see e.g. Vázquez et al. 1996; 2005; and Carraro 2002). After providing evidence that the reddening law is normal, the $Q$-parameter is therefore defined to be:

$Q=(U-B)-0.72 \times(B-V)$.

The value of $Q$ is a function of spectral type and absolute magnitude (Schmidt-Kaler 1982).

This technique permits us to identify bona fide members of spectral type as late as A0V. We have identified 146 stars in our sample with a mean $E(B-V)$ of $0.25 \pm 0.10$ (group A), 96 stars with a mean $E(B-V)$ of $0.52 \pm 0.05$ (group B), and 212 stars with a mean $E(B-V)$ of $1.30 \pm 0.20$ (group $\mathbf{C}$ ).

To obtain an estimate of the mean distance to the three groups, we then used the variable extinction diagram shown in Fig. 6, where to derive $\left(V-M_{V}\right)$ we adopt $M_{V}$ as a function of spectral type from Schmidt-Kaler (1982). Yellow triangles, blue squares, and red circles depict members of groups $\mathbf{A}, \mathbf{B}$, and $\mathbf{C}$, respectively. The four lines plotted have been drawn by adopting the normal extinction law $\left(R_{V}=3.1\right)$, and correspond to four different absolute distance moduli $\left(V_{0}-M_{V}\right)$ : 10.0 (solid), 12.0 (dotted), 14.0 (short-dashed), and 16.0 (long-dashed). The histograms in the right panel of this figure give the apparent distance moduli distribution, from which we infer the mean distance to the three groups.

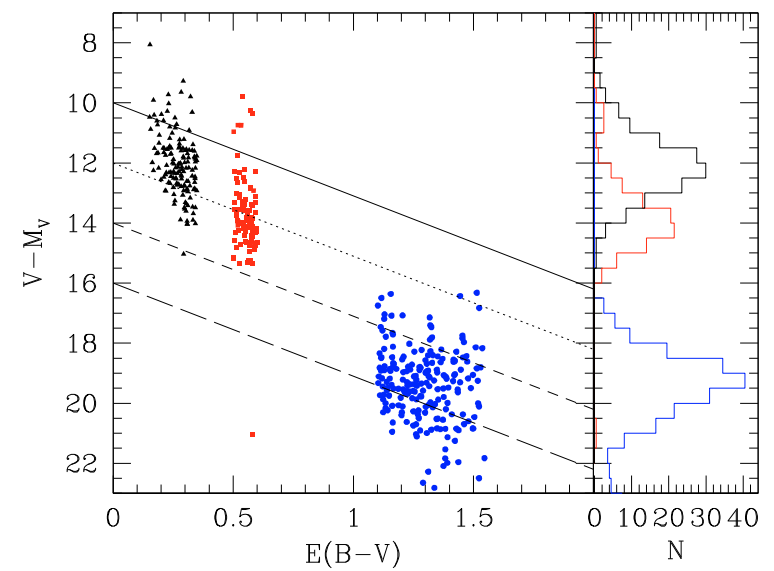

Fig. 6. Variable extinction diagram for all early-type stars for which individual reddening was measured. The four lines plotted have been drawn by adopting the normal extinction law $\left(R_{V}=3.1\right)$, and correspond to four different absolute distance moduli $\left(V_{0}-M_{V}\right): 10.0$ (solid), 12.0 (dotted), 14.0 (short-dash) and 16.0 (long-dash). The histograms in the right panel of this figure indicate the apparent distance moduli distribution, from which we infer the mean distance of the three groups.

One can in fact extrapolate the absolute distance modulus $(m-M)_{V, 0}$ reading the value along the $Y$-axis, where the reddening is zero.

In the left panels of Figs. 7-9 we present $(B-V)$ versus $(U-B)$ color-color diagrams for the stars of groups $\mathbf{A}$ (yellow triangles), B (red squares) and $\mathbf{C}$ (blue circles), respectively. The right panels of the three figures indicate the distribution on the plane of the sky of the objects in each group. In these latter panels, stars have been plotted as circles, which were scaled according to their $V$ magnitude. The fields shown in these figures 

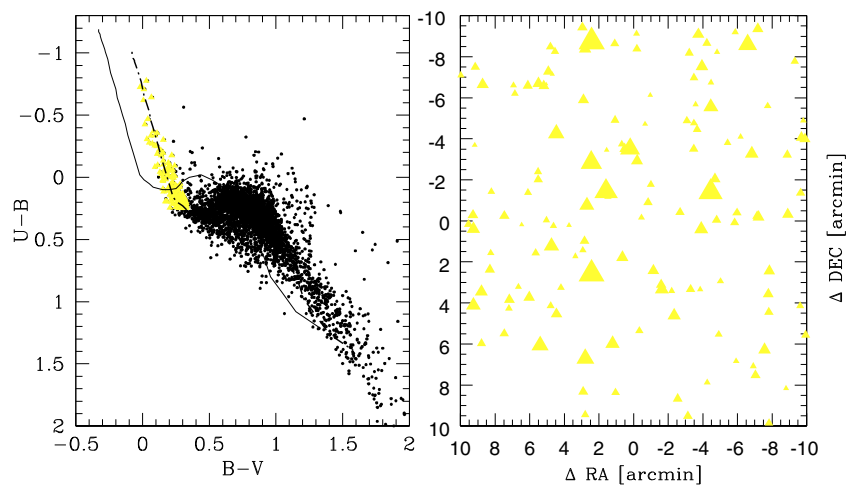

Fig. 7. Stars belonging to group A (yellow triangles). Left panel (as well as left panel of Fig. 6) illustrates how this group was selected on a reddening basis. See text for details.

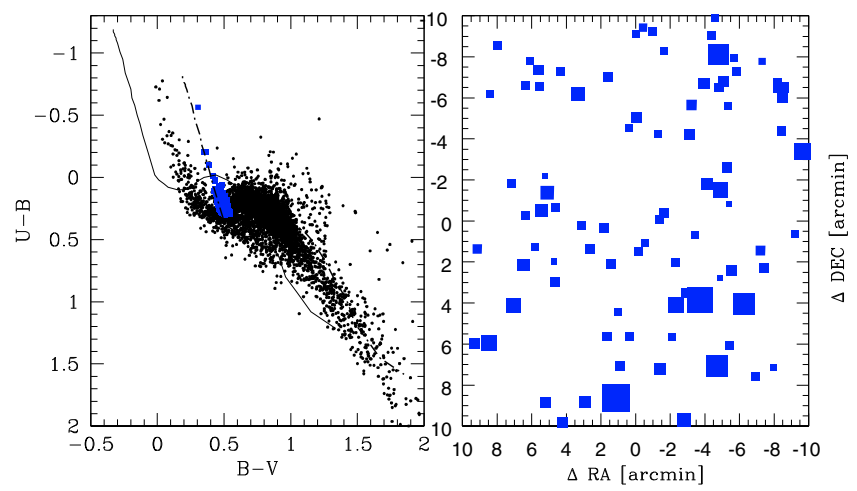

Fig. 8. Same as Fig. 6, for stars belonging to group B (red squares). See text for details.

have been centered at $\mathrm{RA}=11^{\mathrm{h}} 05^{\mathrm{m}} 48.5^{\mathrm{s}}$, Dec $=-61^{\circ} 11^{\prime} 5.9^{\prime \prime}$. North is up, and East to the left as in Fig. 1.

\subsection{Group A}

Inspection of the right panel of Fig. 7 shows that the objects in group A are evenly distributed on the sky, with no evidence of any particular concentration.

The sequence defined by this group in the variable reddening diagram (Fig. 6) has a mean intrinsic distance modulus of $12.0 \mathrm{mag}$, with a large spread. Most of the stars are locateed at a mean distance of $2.5 \pm 1.5 \mathrm{kpc}$. This mean distance is conistent with this group being oart of the Carina branch of the CarinaSagittarius arm closest to the Sun. The observed distance spread is compatible with the typical size of the inner Galactic spiral arms (1.5-2.0 kpc, Bronfman et al. 2000).

We therefore conclude that group A consists of young field stars belonging to the Carina-Sagittarius arm, confirming the suggestion by Sh04. We dispute, however, the proposal that these stars form a star cluster as suggested by Shorlin (1998), whose conclusion was possibly the result of the small FOV covered by their photometry. Our wider area coverage clearly enables us to see that are looking at a population of field stars of same reddening and age, but located at different position inside the Carina arm.

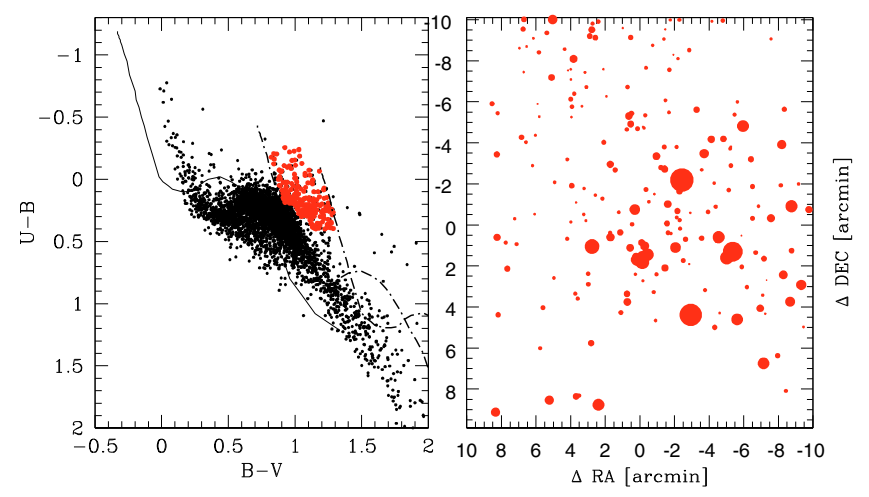

Fig. 9. Same as Fig. 6, for stars belonging to group $\mathbf{C}$ (blue circles). See text for details.

\subsection{Group B}

As was the case for group $\mathbf{A}$, the objects in group $\mathbf{B}$ are evenly distributed on the plane of the sky, but are, on average, fainter (see right panel of Fig. 8).

In the variable-reddening diagram (Fig. 6), they trace a sequence of stars larger distances than those of group $\mathbf{A}$. They have a mean absolute distance modulus of $14.0 \mathrm{mag}$, which implies a bulk distance of $6.0 \pm 2.0 \mathrm{kpc}$. The stars in this group are also young, and, according to modern descriptions of the MW spiral structure (Vallée 2005; Russeil 2003), they are most probably located in the part of the Carina branch more distant from the Sun. The tangent to the Carina-Sagittarius arm points to $l \sim 280^{\circ}$, in suc a way that the line-of-sight to these groups is expected to cross the Carina portion of this arm twice.

This group had not been identified before, which may explain the conflicting conclusions reached by Sh04 and Wa05 about the location and distance of the putative star cluster associated with WR38 and WR38a (see discussion below).

\subsection{Group $C$}

This is the most interesting group of faint, young stars with common reddening. Our mean $E(B-V)$ value $(1.30 \pm 0.20)$ is lower than the value measured by Sh04, but still marginally consistent within the errors declared. The 212 stars extracted within this reddening range have a broad distance distribution (see Fig. 6), are faint, and are fairly evenly distributed across the field of view (see Fig. 9). Careful inspection of Fig. 9 indicates the existence of a concentration of stars close to the center of the field. This concentration corresponds to Shorlin 1 (C1104-610a, Sh04).

We now ask whether this is really a cluster or only a chance concentration of bright stars along the line-of- sight and what its location is exactly. As Sh04 argued, the reality of the cluster can be assessed only with far deeper photometry. To address these two issues, we use the variable-reddening diagram (Fig. 6) and he CMDs presented in Figs. 10 and 11, compiled for the objects belonging to group $\mathbf{C}$ with the the highest reddening $(E(B-V)>1.45)$, such as Shorlin 1 (see Sh04). Filled symbols in Figs. 10 and 11 indicate stars within a 0.6' wide circle centered on Shorlin 1 (nominal center RA $=11^{\mathrm{h}} 05^{\mathrm{m}} 46.52^{\mathrm{s}}$; Dec $\left.=-61^{\circ} 13^{\prime} 49.1^{\prime \prime}\right)$, which is the zone in which the cluster shappens to be located.

Given that these common reddening stars of group $\mathbf{C}$ are distributed evenly in the field (see Fig. 9), we can compare the position (in a CMD) of objects in the general field with that of objects in the Shorlin 1 region. As shown by the CMDs presented in Fig. 10, general field stars (open symbols) occupy the same 


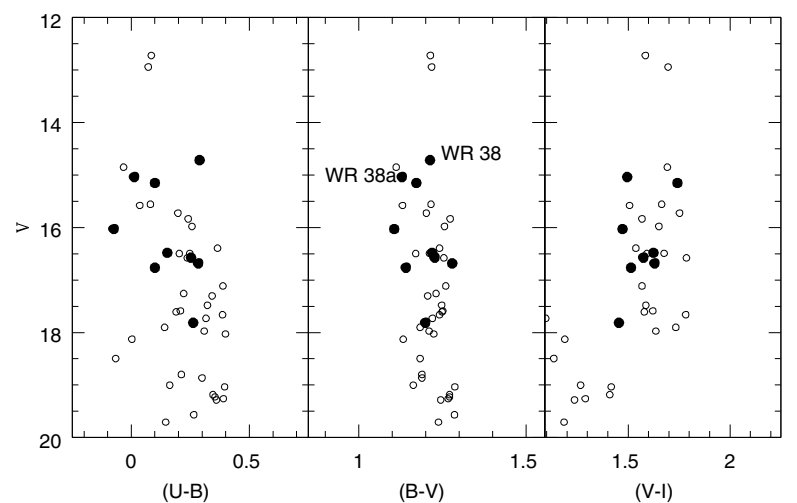

Fig. 10. Color-magnitude Diagrams for stars belonging to group $\mathbf{C}$, for different color combinations. Filled symbols indicate stars inside the Shorlin 1 area.

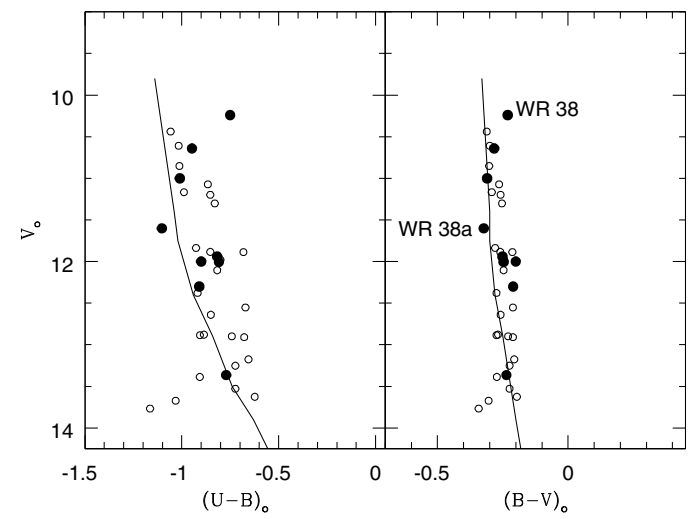

Fig. 11. Reddening-free color-magnitude Diagram for stars belonging to group $\mathbf{C}$, for different color combinations. Filled symbols indicate stars inside Shorlin 1 area. In this figure only stars having $E(B-V)$ larger than 1.45 are plotted. See text for more details.

region as Shorlin 1 stars (filled symbols). This suggests that they have similar distances and ages, and represent a common, evenly distributed, population.

The same conclusion can be drawn from the reddening-free CMDs presented in Fig. 11. In these plots, the stars within the candidate cluster area and those in the general field, define a similar sequence. The $V_{0}$ versus $(B-V)_{0}$ CMD presented in the right panel of Fig. 11 has been plotted to the same scale as Fig. 5 of Sho04. In spite of our photometry being six magnitudes deeper in $V$, we note that we only detect one fainter object in the cluster area. We can therefore be confident that there are no fainter stars associated with the cluster.

The ZAMS superimposed in the both panels of Fig. 11 represents $(m-M)_{V, 0}=15.5$, which suggests that the distance of group $\mathbf{C}$ is about $12.7 \pm 3.0 \mathrm{kpc}$. Both Shorlin 1 members and field stars however exhibit a significant distance spread (see Fig. 6).

For the reasons summarized below, we conclude that there is no clear evidence of a star cluster at the location of WR38/WR38a.

- A cluster hosting two WR stars would have to be a relatively massive one, with numerous stars of spectral type later than $B$, which is in conflict with what we see;

- we do not see any significant concentration of stars at the putative cluster location (see Fig. 1);
Table 3. Properties of the three distinct groups that we detected in the field surrounding WR 38 and WR 38a.

\begin{tabular}{lcccc}
\hline \hline Group & $E(B-V)$ & $d_{\odot}$ & Age & Note \\
\hline A & $0.25 \pm 0.10$ & $2.5 \pm 1.5$ & $<100 \mathrm{Myr}$ & Carina \\
B & $0.52 \pm 0.05$ & $6.0 \pm 2.0$ & $<100 \mathrm{Myr}$ & Carina \\
C & $1.30 \pm 0.20$ & $12.7 \pm 3.0$ & $<100 \mathrm{Myr}$ & Perseus \\
\hline
\end{tabular}

- the sequence defined by Shorlin 1 in the CMDs is also occupied by field stars, which have the same reddening, and are evenly distributed in the field (see Figs. 9-11);

- the stars defined to be members of the cluster (the 8 stars discovered by Sh04, plus one fainter object from this work) exhibit a significant distance spread (see Fig. 6), which is inconsistent with a it being, even a loose, star cluster.

To summarize our findings, we report in Table 3 the main properties of the three groups. For the age we only provide an upper limit, since the populations under investigations are certainly young, and a precise age estimate, which will be in all cases difficult, is beyond the scope of this work.

\section{Conclusions}

The large angular coverage and depth, of our $U B V I$ photometry in the region about the WR38/WR38a has allowed us to identify the nature of the stellar populations towards Galactic longitude $\approx 290^{\circ}$, in the fourth Galactic quadrant.

Our results can be visualized with the aid of Fig. 12, where the spiral structure of the Galaxy (Vallée 2005) is presented in the $X-Y$ plane, where $X$ points in the direction of Galactic rotation and $Y$ points towards the anti-center. The Carina and Perseus arms are indicated by the symbols $I$ and $I I$, respectively. The Galactic center and position of the Sun are also indicated, at $(0.0,0.0)$ and $(0.0,8.5)$, respectively. The position, pitch angle, and extension of the arms are clearly model dependent to a lesser extent for the Carina arm (which is well known) and significantly more for the less well-known Perseus arm (although both HI observations (Levine et al. 2006) and HII observations (Russeil 2003) agree in terms of the approximate location and extent of the Perseus arm). Beyond $\sim 9 \mathrm{kpc}$ from the Sun, we do not expect to find spiral features related to the Carina arm (Georgelin et al. 2000), although more distant structures can be associated with the Perseus arm. According to Levine et al. (2006, their Fig. 4), at $l=290^{\circ}$ the Perseus arm is located in the range $-18.0 \leq X \leq-13.0$ and $0.0 \leq Y \leq 2.0 \mathrm{kpc}$, respectively, which agrees well with the Vallée model.

By a dashed arrow, we indicate the line-of-sight in the direction of our field, and by thick segments, the distance range that we derived for the three groups $(\mathbf{A}, \mathbf{B}$, and $\mathbf{C})$ identified in this Galactic direction (see also Table 3). Figure 12 illustrates how the groups detected correlate well with the position of the Carina and Perseus arm. This confirms Sh04's suggestion that the most distant group $(\mathbf{C})$ is probably associated with the extension of the Perseus arm in the fourth Galactic quadrant, and represents the first optical detection of this arm. However, in contrast to Sh04, we propose that this extreme group is not a star cluster, but instead a group of young stars uniformly distributed within the Perseus arm.

The line-of-sight we investigateis about $\sim 1^{\circ}$ below the Galactic plane. At distances of 2.5, 6.0, and $12.7 \mathrm{kpc}$ this implies heights below the Galactic plane of about 50, 100, and $210 \mathrm{pc}$, respectively. These are sizeable values, which reflect the 


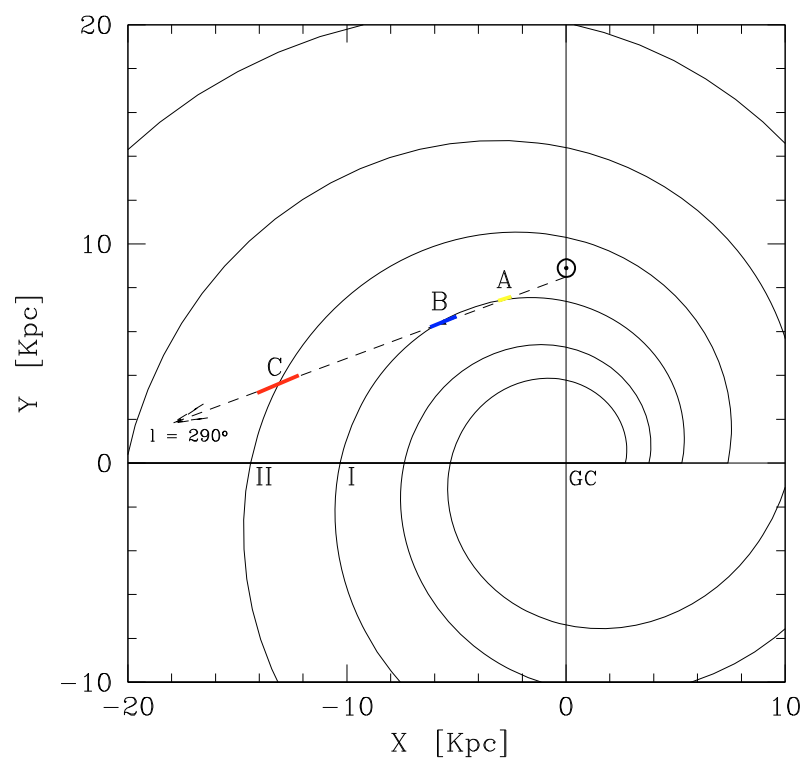

Fig. 12. Schematic view of the spiral structure of the Galaxy (Vallee 2005). $X$ indicates the direction of Galactic rotation, and $Y$ represents the distance to the anti-center. The position of the Sun $(0.0,8.5)$ and the Galactic center $(0.0,0.0)$ are indicated. The symbols I and II indicate the Carina and Perseus spiral arms, respectively. Thick segments illustrate the location of the three groups $(\mathbf{A}, \mathbf{B}$ and $\mathbf{C})$ discussed in the text. See text for details.

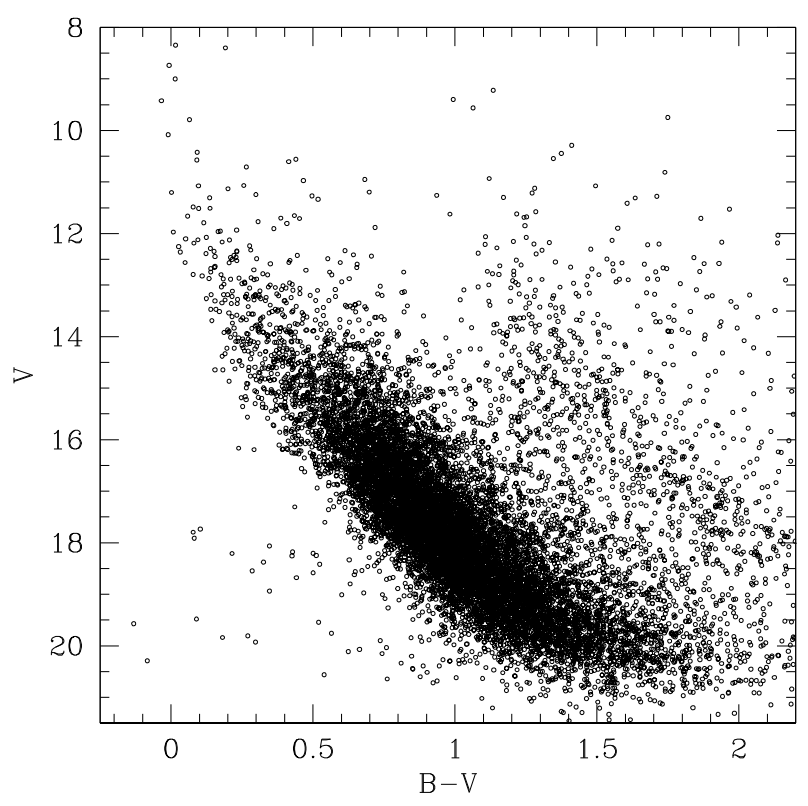

Fig. 13. CMD of all stars with $B V$ photometry in the field of study.

presence of the Galactic warp in this zone of the disk (Momany et al. 2006).

We finally discuss our photometric database and possible detection of the Argus overdensity discussed by Rocha-Pinto et al. (2006), or the Monoceros Ring, which was proposed to be associated with Shorlin 1 (Frinchaboy et al. 2004). In Fig. 13, we present $V$ versus $(B-V)$ CMD diagram for all the stars with $B V$ photometry.

The distribution of stars in this diagram is relatively easy to describe. Apart from the prominent blue sequence of young stars brighter than $V \approx 14.0$, which are the objects of $\mathbf{A}$, we can distinguish two other remarkable features. The first is a thick Main Sequence (MS) towards magnitudes fainter than $V \approx$ 14.0, widened by photometric errors and binaries, but reflecting mostly the fact we are sampling objects at different distances, and with different reddening, in the Galactic disk. Along this thick MS, we do not find any evidence of a blue Turn Off Point, typical of the metal-poor, intermediate-age, population of the Monoceros Ring (Conn et al. 2007). We therefore conclude that there is no indication of the presence of the Monoceros Ring or the Argus system in our field. The second remarkable feature is a Red Giant Branch, significantly widened and somewhat skewed by variable reddening, which consists of giant stars at different distances. The relatively high number of giants can be easily accounted for, because we are sampling a field located $1^{\circ}$ below the Galactic plane.

Our results confirm the effectiveness of multicolor optical photometry in the study of the structure of the MW disk. More fields need to be observed to be able to constrain the spiral structure in the fourth Galactic quadrant, in particular the shape and extent of the Perseus arm, and, possibly, detect the more distant Norma-Cygnus arm.

In constrast to radio observations, which must rely on the poorly known Galactic rotation curve, optical observations, particularly in directions of low absorption, can constrain the distance to spiral features with superior accuracy.

Acknowledgements. Tomer Tal and Jeff Kenney are thanked sincerely for securing part of the observations used in this paper. G.C. acknowledges R. A. Vázquez for very fruitful discussions. E.C. acknowledges the Chilean Centro de Astrofísica FONDAP (No. 15010003).

\section{References}

Arias, E. F., Charlot, P., Feissel, M., \& Lestrade, J.-F. 1995, A\&A, 303, 604 Benjamin, R. A. 2008, in Massive Star Formation: Observations Confront Theory, ASP Conf. Ser., 387, 375

Bronfman, L., Casassus, S., May, J., \& Nyman, L.-Å. 2000, A\&A, 358, 521 Carraro, G. 2002, MNRAS, 331, 785

Carraro, G., Vázquez, R. A., Moitinho, A., \& Baume, G. 2005, ApJ, 630, L153

Conn, B. C., Lane, R. R., Lewis, G. F., et al. 2007, MNRAS, 376, 939

Dean, J. F., Warren, P. R., \& Cousins, A. W. J. 1978, MNRAS, 183, 569

Frinchaboy, P. M., Majewski, S. R., Crane, J. D., et al. 2004, ApJ, 603, L21

Georgelin, Y. M., Russeil, D., Amram, P., et al. 2000, A\&A, 357, 308

Johnson, H. L. 1966, ARA\&A, 4, 193

Landolt, A. U. 1992, AJ, 104, 372

Luna, A., Bronfman, L., Carrasco, L., \& Maj, J. 2006, ApJ, 641, 938

Levine, E. S., Bliz, L., \& Heiles, C. 2006, Science, 312, 1773

Moitinho, A., Vázquez, R. A., Carraro, G., et al. 2006, MNRAS, 368, L77

Moitinho, A., Vázquez, R. A., \& Carraro, G. 2008, A\&A, to be submitted

Martin, N. F., Ibata, R. A., Bellazzini, M., et al. 2004, MNRAS, 348, 12

Momany, Y., Zaggia, S., Gilmore, G., et al. 2006, A\&A, 451, 515

Patat, F., \& Carraro, G. 2001, MNRAS, 325, 1591

Rocha-Pinto, H. J., Majewski, S. R., Skrutskie, M. F., et al. 2006, ApJ, 640, L147

Russeil, D. 2003, A\&A, 397, 133

Schmidt-Kaler, Th. 1982, Landolt-Börnstein, Numerical data and Funct ional Relationships in Science and Technology, New Series, Group VI, Vol. 2(b), ed. K. Schaifers, \& H. H. Voigt (Berlin: Springer Verlag), 14

Shorlin, S. L. 1998, M.Sc. Thesis, Saint Mary's University

Shorlin, S. L., Turner, D. G., \& Pedreros, M. 2004, PASP, 116, 170 (Sh04)

Stetson, P. B. 1987, PASP, 99, 191

Tapia, M., Roth, M., Vázquez, R. A., \& Feinstein, A. 2003, MNRAS, 339, 44

Vallée, J. P. 2005, AJ, 130, 569

Vázquez, R. A., Baume, G. L., Feinstein, C., \& Prado, P. 1996, A\&A, 116, 75

Vázquez, R. A., Baume, G. L., Feinstein, C., Nun̈ez, J. A., \& Vergne, M. M. 2005, A\&A, 430, 471

Vázquez, R. A., May, J., Carraro, G., et al. 2008, ApJ, 672, 930

Wallace, D. J., Gies, D. R., Moffat, A. F. J., Shara, M. M., \& Niemela, V. 2005, AJ, 130, 126 (Wa05)

Yanny, B., Newberg, H. J., Grebel, E. K., et al. 2003, ApJ, 588, 824 